\title{
Radiomics-based method for diagnosis of calciphylaxis in patients with chronic kidney disease using computed tomography
}

\author{
Qian Yu ${ }^{1}$, Yuqiu Liu ${ }^{2}$, Xiaotong Xie ${ }^{2}$, Jinqiang Liu ${ }^{1}$, Shan Huang ${ }^{1}$, Xiaoliang Zhang ${ }^{2}$, Shenghong Ju ${ }^{1}$ \\ ${ }^{1}$ Department of Radiology, Jiangsu Key Laboratory of Molecular and Functional Imaging, Zhongda Hospital, Medical School, Southeast University, \\ Nanjing, China; ${ }^{2}$ Institute of Nephrology, Zhongda Hospital, Medical School, Southeast University, Nanjing, China
}

Contributions: (I) Conception and design: S Ju, X Zhang; (II) Administrative support: S Ju, X Zhang; (III) Provision of study materials or patients: Q Yu, Y Liu, S Huang; (IV) Collection and assembly of data: Q Yu, X Xie, J Liu, S Huang; (V) Data analysis and interpretation: Q Yu; (VI) Manuscript writing: All authors; (VII) Final approval of manuscript: All authors.

Correspondence to: Shenghong Ju, MD, PhD. Professor of Medicine, Department of Radiology, Jiangsu Key Laboratory of Molecular and Functional Imaging, Zhongda Hospital, Medical School, Southeast University, 87 Ding Jia Qiao Road, Nanjing, China. Email: jsh0836@hotmail.com.

\begin{abstract}
Calciphylaxis is a rare, life-threatening condition that affects patients with chronic kidney disease (CKD) undergoing dialysis. Skin biopsy as the gold standard causes ulceration, bleeding, or infection. This study aimed to develop radiomic methods using CT as a noninvasive method for calciphylaxis diagnosis. The confirmed calciphylaxis patients (Group I), pathologically confirmed non-calciphylaxis patients (Group II), and CKD patients (Group III) from October 1, 2017, to November 30, 2019, were enrolled. Training: 70\% of patients of Group I and all Group III. Test: 30\% of patients of Group I and all Group II. ROI was set at the skin lesion including the soft tissue. First-order and texture features were extracted from each lesion unit. CT-based radiomic models were on the basis of logistic regression (LR) and support vector machine (SVM). Additionally, model performance was evaluated in the test dataset and compared with the plain radiography and bone scintigraphy. In total, 124 lesions and 38 lesions were identified in training and test datasets. Radiomic models were effective in detecting calciphylaxis in patients with CKD, with AUCs of 0.93 (95\% CI: $0.924-0.953$ ) and 0.93 (95\% CI: 0.921-0.953) (SVM and LR) in test. The SVM model manifested a sensitivity and specificity of 0.89 and 0.8 , and 0.78 and 0.90 , at high-sensitivity and high-specificity operating points, respectively. Similar performance was found in the LR model. Radiomic models were more effective than plain radiography and bone scintigraphy (Delong test, $\mathrm{P}<0.05$ ). Verification studies showed the features which manifested the real variability of lesions. In this research, it primarily developed a radiomic method for noninvasive detection of calciphylaxis in patients with CKD. Through this method, calciphylaxis can be detected when invasive procedures are not feasible.
\end{abstract}

Keywords: Calciphylaxis; computed tomography (CT); radiomics

Submitted Oct 28, 2020. Accepted for publication May 10, 2021.

doi: $10.21037 /$ qims-20-1211

View this article at: https://dx.doi.org/10.21037/qims-20-1211

\section{Introduction}

Calciphylaxis is a rare, life-threatening condition with high morbidity and mortality, commonly affecting patients with chronic kidney disease (CKD) undergoing dialysis, with death caused mainly by sepsis secondary to infected ulcers (1).
Calciphylaxis diagnosis is challenging, for which skin biopsy is a gold standard. Nonetheless, it increases the risk of ulceration, bleeding, and necrosis owing to deep incisions (2). A noninvasive tool for diagnosing calciphylaxis lesions is beneficial for patients with skin lesions and CKD.

The potential usefulness of noninvasive radiology, 
including plain radiography and bone scintigraphy, has been reported, whereas the usefulness of computed tomography (CT) has not been assessed (3-5). We hypothesized that histological changes, covering vessel calcification, thrombosis, interstitial calcium deposits, and adipose tissue remodelling, in the subcutaneous tissue of calciphylaxis lesions $(1,2,6-8)$ would be reflected in the CT value and texture adopting the radiomic method, which is a highthroughput approach using image features calculated from histogram and texture analysis $(9,10)$.

Hence, this research aims to develop and test a CT-based radiomic model for noninvasive detection of calciphylaxis in patients with CKD.

\section{Methods}

\section{Study population}

This retrospective, cohort study was conducted in accordance with the Declaration of Helsinki (as revised in 2013) and was approved by the local institutional ethics review board. Additionally, the need for written informed consent from the participants was waived.

In this research, it retrospectively reviewed the institutional datasets for patients with the initial diagnosis of calciphylaxis from October 1, 2017, to November 30, 2019. Calciphylaxis diagnosis protocol was based on "Criteria for Diagnosis of Calciphylaxis" illustrated by McCarthy et al. (11) One patient with risk factors and clinical conditions would undergo histopathological examination and receive multidisciplinary discussion involving nephrology, dermatology, radiology, and pathology departments with experienced doctors $(12,13)$. If one is finally diagnosed with calciphylaxis as revealed in the histopathological result, he or she was included as calciphylaxis patients (Group I). If one with possible calciphylaxis but finally ruled out by histopathology, he or she was considered as suspected calciphylaxis patients (pathologically confirmed noncalciphylaxis patients) (Group II). Viscera calciphylaxis was not included in the analysis.

In the next step, CT scan data involving lesion location was searched. For instance, if one patient had ulceration in the right leg, the researcher would find lower extremities' non-contrast CT images in Picture Archiving and Communication Systems. All CT scan followed routine CT scan protocols.

Nevertheless, for limited negative cases, based on the hypothesis, Patients with CKD underwent dialysis during the same time period with abdominal CT, who displayed no features of calciphylaxis comprised the non-calciphylaxis cohort (CKD-non-calciphylaxis) (Group III), so as to extract radiomics features about subcutaneous tissue.

The training cohort comprised $70 \%$ of patients randomly selected from those with calciphylaxis and all non-calciphylaxis patients with CKD.

The independent test cohort consisted of the remaining $30 \%$ of patients with calciphylaxis and all patients suspected of having calciphylaxis. There was no overlapping between training and test data.

\section{Radiomic analysis and model building}

Figure 1 displays the pipeline of the radiomic model with the feature extraction and model building on each lesion unit.

\section{Lesion segmentation}

The CT images containing the lesion patches were segmented using 3Dslicer (version 4.10.0; https://www. slicer.org/) (Figure 1). As determined by an experienced nephrologist and radiologist based on photos or records, a $50 \times 50 \times 50 \mathrm{~mm}^{3}$ box was placed in the area of the lesion for patients with calciphylaxis and those suspected of calciphylaxis. For CKD-non-calciphylaxis patients, 2 boxes of the same size were placed at the L3 vertebra of their backs. Apart from muscle and bone in the box, all subcutaneous tissues would be segmented as lesion patches.

\section{Feature extraction}

In the first step, all images and masks were resampled to form isotropic voxels of unit dimension with 1 voxel corresponding to $1 \mathrm{~mm}^{3}$ to guarantee comparability. By centring the image at the mean with a standard deviation and re-charting the histogram to conform to $1 \pm 3 \mathrm{r}$ (l: the average grey level within the VOI; r: the grey-level standard deviation), image normalization was realised.

For each lesion, there were a total of 774 features. In accordance with pyradiomics, both first-order and textural features were calculated from the original images and those with the wavelet filter (14).

\section{Model building}

Normalisation was applied to the feature matrix with each vector featured with a zero centre and standard deviation.

To reduce the space dimensionality of the feature, the Pearson correlation coefficient (PCC) of the feature pair 

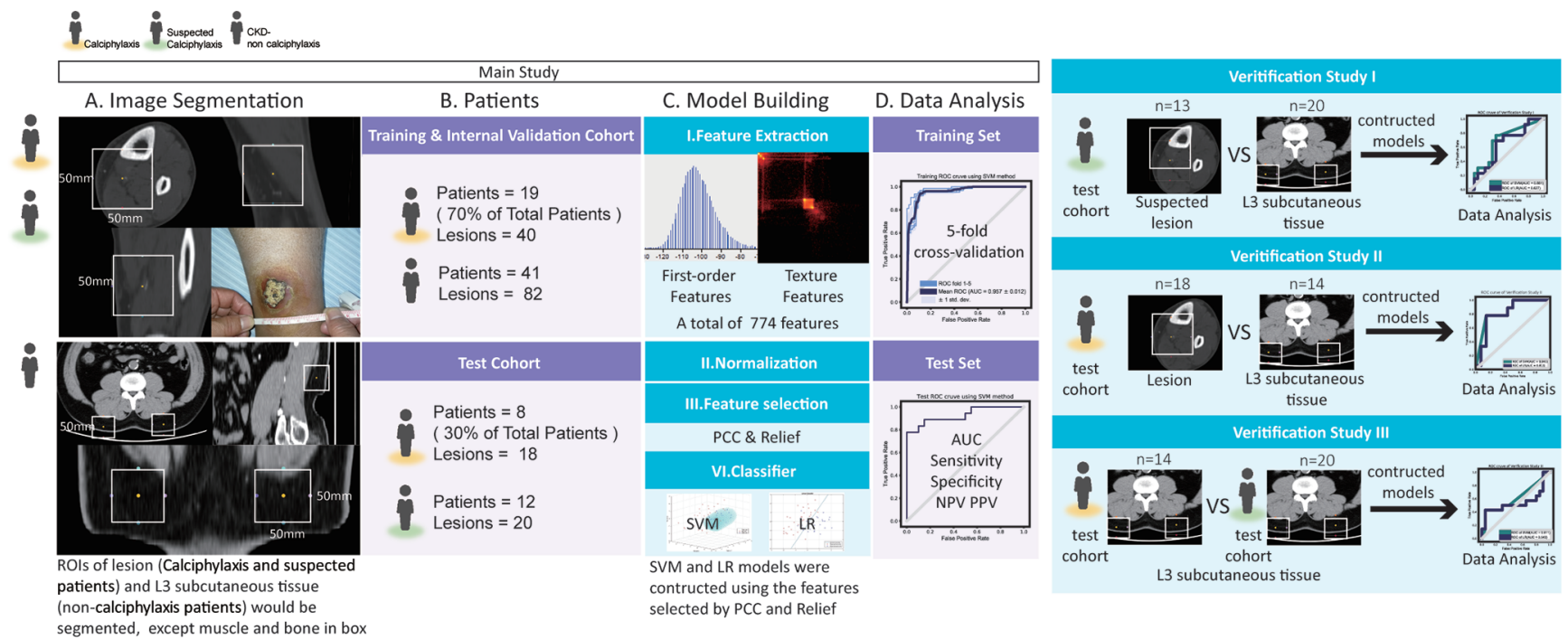

Figure 1 Flow chart of main study and verification studies. AUC, areas under the ROC curves; CKD, chronic kidney disease; LR, logistic regression; SVM, support vector machine; ROI, region of interest; PPV, positive predictive value; NPV, negative predictive value; ROC, receiver operating characteristic; PCC, Pearson correlation coefficient.

was adopted. Subsequently, Relief was employed to identify the features most closely correlated with the outcome, selecting the sub dataset and finding relative features according to the label by recursion.

The model and the robustness of the extracted features were built and verified, respectively, through two different supervised learning algorithms, namely the support vector machine (SVM) and the logistic regression (LR) (15). The former searches the hyper-plane to label the cases by mapping the features in a higher dimension, whereas the latter is deemed as a linear classifier via penalised maximum likelihood. Moreover, the linear kernel was used for explicable feature coefficients in the SVM model. In the training course, the 5 -fold cross-validation of the training dataset was conducted to confirm the model's performance.

\section{Model performance and stability}

\section{High specificity and sensitivity points}

In line with the method described in Chilamkurthy et al. (16), high specificity and sensitivity points were selected from the receiver operating characteristic (ROC) curve of the training dataset.

\section{Model performance}

The test dataset was adopted for the performance evaluation of the model. More specifically, areas under the ROC curves (AUCs), as well as the sensitivity, specificity, negative predictive value (NPV), and positive predictive value (PPV), at these 2 operating points were recorded.

Since the diagnostic performance of plain radiography (presence of vascular calcification in lesion peripheries) and bone scintigraphy in the test dataset was assessed, the AUCs of the radiomic model were compared with those of other radiological methods through the Delong test.

\section{Verification studies}

To determine the ability of the model to identify the calciphylaxis lesion in CKD patients, rather than classify the extremities and abdomen tissue: (I) this research did not extract shape-based features and designed three verification studies. (II) prior to study, it researched the abdomen CT scan at the time of initial diagnosis in the test dataset.

For calciphylaxis patients, 7 patients had abdomen CT scan without skin lesions at back or abdomen, and a total of 14 patches segmented manifested in Figure 1. For suspected calciphylaxis patients, 7 of 12 patients (13 of 20 lesions) were not at the back or abdomen, and they had an abdomen CT scan at the time of initial diagnosis. Another three suspected patients with abdomen CT scan were covered as well. Consequently, a total of $20(7 \times 2+3 \times 2)$ abdomen subcutaneous patches were segmented in suspected calciphylaxis patients (Figure 1).

Study I: there was a comparison between the results 
of the suspected lesion and L3 subcutaneous tissue in suspected calciphylaxis patients.

Study II: there was a comparison between skin lesion and L3 subcutaneous tissue in calciphylaxis patients. Patients with skin lesion in the back would be excluded.

Study III: there was a comparison between L3 subcutaneous tissue in calciphylaxis patients and suspected calciphylaxis patients.

\section{Statistical analysis}

In order to assess the patients' clinical and demographic characteristics, the Chi-square test (or the Fisher's exact test if necessary) was conducted to assess categorical variables, while the Mann-Whitney test was used for evaluating continuous variables in SPSS (version 22.0. IBM Crop. Armonk, NY, USA). Then, feature selection and model establishment were realised through FeAture Explorer (FAE, v0.2.5, https://github.com/salan668/FAE) on Python (3.6.8, https://www.python.org/). Furthermore, SPSS and Python were employed to calculate test values including the AUCs with $95 \%$ confidence interval (CI), the sensitivity, and the specificity. The Delong test was performed using the "pROC" package (R language 3.0.2, R Core Team, 2013), and a statistically significant $\mathrm{P}$ value should be smaller than 0.05 .

\section{Results}

\section{Patient characteristics}

In this retrospective study, 32 patients diagnosed with calciphylaxis were enrolled in line with the histopathological result in Group I, and 15 patients suspected of having calciphylaxis in Group II, and finally, 27 and 12 patients with non-contrast CT scans containing skin lesions were enrolled in radiomics analysis respectively. Moreover, noncontrast CT scans containing skin lesions were included. Nevertheless, for limited negative cases, as shown in the hypothesis, 41 patients with CKD, undergoing dialysis during the same time period with abdominal CT, were enrolled in the non-calciphylaxis cohort (CKD-noncalciphylaxis) (Group III).

The training cohort comprised 70\% (19/27) of patients with 40 lesions randomly selected from Group I and Group III (41 patients with 82 image patches). The independent test cohort comprised the rest $30 \%(8 / 27)$ of patients with calciphylaxis and Group II (12 patients with 20 lesions). Details about additional sample size consideration are presented in the Appendix 1.

Baseline demographic and clinical characteristics of patients are outlined in Table 1. No dramatic differences existed in all baseline characters $(\mathrm{P}>0.05)$. The location of the skin lesions and CT scan of patients in Group I and II are listed in Table 2.

\section{Performance of the radiomic model}

The model, based on 8 features including 4 first-order features and 4 textural features, showed the highest AUC on the validation data set. Significant features and coefficients utilised in the models are manifested in Table S1.

Figure 2 and Table 3 conclude the performance of the radiomic model with the SVM and LR methods. In the training and test dataset, the model based on SVM and LR methods demonstrated the highest performance compared with conventional imaging methods. AUCs of both models reached 0.93 (0.924-0.953) (SVM) and 0.93 (0.921-0.953) (LR) in the test dataset. At the high-sensitivity point, the SVM and LR model revealed diagnostic performance for diagnosis of calciphylaxis with the sensitivity, specificity, PPV and NPV of 0.89, 0.80, 0.80, 0.89 and 0.89, 0.85, $0.84,0.90$ respectively. At the high-specificity point, the SVM and LR model showed diagnostic performance for diagnosis of calciphylaxis with the sensitivity, specificity, PPV and NPV of $0.78,0.90,0.88,0.82$ and $0.78,1.0,1.0$, 0.83 respectively.

In a subset of the test dataset, where patients had plain radiograph and bone scintigraphy, LR performed the highest AUC, followed by the SVM model (Table 4). Radiomics models illustrated higher AUCs than plain radiograph and bone scintigraphy (Delong test, $\mathrm{P}<0.05$, Table S2). Bone scintigraphy also displayed a high specificity in the diagnosis of calciphylaxis.

\section{Results of verification studies}

As shown in Figure 3, in study I and III, the models on SVM and LR cannot classify (I) suspected lesions and L3 subcutaneous tissue in patients with suspected calciphylaxis and (II) L3 subcutaneous tissue in patients with confirmed calciphylaxis and that in patients with suspected calciphylaxis according to low AUCs (0.543-0.681). Despite this, in study II, the model with AUCs of 0.84 and 0.81 (SVM and LR respectively) performed better in the classification of skin lesions and L3 subcutaneous tissue in patients with calciphylaxis (Figure 3). 
Table 1 Characteristics of patients

\begin{tabular}{|c|c|c|c|c|c|c|}
\hline Characteristic & \multicolumn{3}{|c|}{ Training cohort } & \multicolumn{3}{|c|}{ Test cohort } \\
\hline Patients, $\mathrm{n}$ & 19 & 41 & & 8 & 12 & \\
\hline Age, mean (SD), years & $49.16(13.45)$ & $63.20(15.21)$ & 0.001 & $66.88(13.22)$ & $59.83(12.64)$ & 0.30 \\
\hline Sex, No. [\%] & $19[100]$ & $41[100]$ & 0.27 & $8[100]$ & 12 [100] & 0.62 \\
\hline Female & $6[32]$ & $21[51]$ & & $1[12]$ & 3 [25] & \\
\hline $\mathrm{BMI}$, mean [SD], $\mathrm{kg} / \mathrm{m}^{2 \mathrm{a}}$ & $22.36[4.10]$ & $22.86[3.62]$ & 0.48 & $23.62[3.41]$ & $23.81[50.02]$ & 0.90 \\
\hline CKD level, No. [\%] & $19[100]$ & $41[100]$ & 0.30 & $8[100]$ & 12 [100] & - \\
\hline 4 & $0[0]$ & $4[10]$ & & $0[0]$ & $0[0]$ & \\
\hline Alcohol abuse, No. [\%] & $0[0]$ & $0[0]$ & - & $0[0]$ & $0[0]$ & - \\
\hline Coronary artery disease, No. [\%] & $1[5]$ & 8 [20] & 0.25 & $1[13]$ & $2[17]$ & $>0.99$ \\
\hline Diabetes, No. [\%] & $4[21]$ & $21[51]$ & 0.03 & $4[50]$ & $5[42]$ & $>0.99$ \\
\hline Diabetes mellitus type 1, No. [\%] & $1[5]$ & $1[2]$ & & $0[0]$ & $0[0]$ & \\
\hline Diabetes mellitus type 2, No. [\%] & $3[16]$ & 20 [49] & & $4[50]$ & $5[42]$ & \\
\hline Hepatobiliary disease, No. [\%] & $4[21]$ & $4[10]$ & 0.25 & 3 [38] & $1[8]$ & 0.26 \\
\hline Hypertension, No. [\%] & 15 [79] & $37[90]$ & 0.25 & $7[88]$ & $11[92]$ & $>0.99$ \\
\hline Hyperparathyroidism, No. [\%] & 7 [367] & $4[10]$ & 0.03 & $3[38]$ & 3 [25] & 0.64 \\
\hline
\end{tabular}

${ }^{\mathrm{a}} 3$ patients in training cohort with no available BMI data, and 1 patient in test cohort with no available BMI data. BMI, body mass index; CKD, chronic kidney disease; SD, standard deviation.

Table 2 Location of skin lesions and CT scan of calciphylaxis and suspected calciphylaxis patients

\begin{tabular}{lll}
\hline Patients & Location of lesions & Site of CT \\
\hline Calciphylaxis patients & Bilateral ankle & \\
1 & Lower extremities & Lower extremities \\
2 & Bilateral hands, Lower extremities, back & lower and upper extremities \\
3 & Lower extremities & Chest, Abdomen, Lower extremities \\
4 & Lower extremities & Chest, Abdomen, Lower extremities \\
5 & Bilateral hands, Lower extremities & Abdomen, Lower extremities \\
6 & Lower extremities & Chest, Abdomen, Lower extremities \\
7 & Lower extremities & Lower extremities \\
8 & Lower extremities & Chest, Lower extremities \\
9 & Lower extremities, right foot & Chest, Abdomen, Lower extremities \\
10 & Bilateral feet & Chest, Abdomen, Lower extremities \\
11 &
\end{tabular}

Table 2 (continued) 
Table 2 (continued)

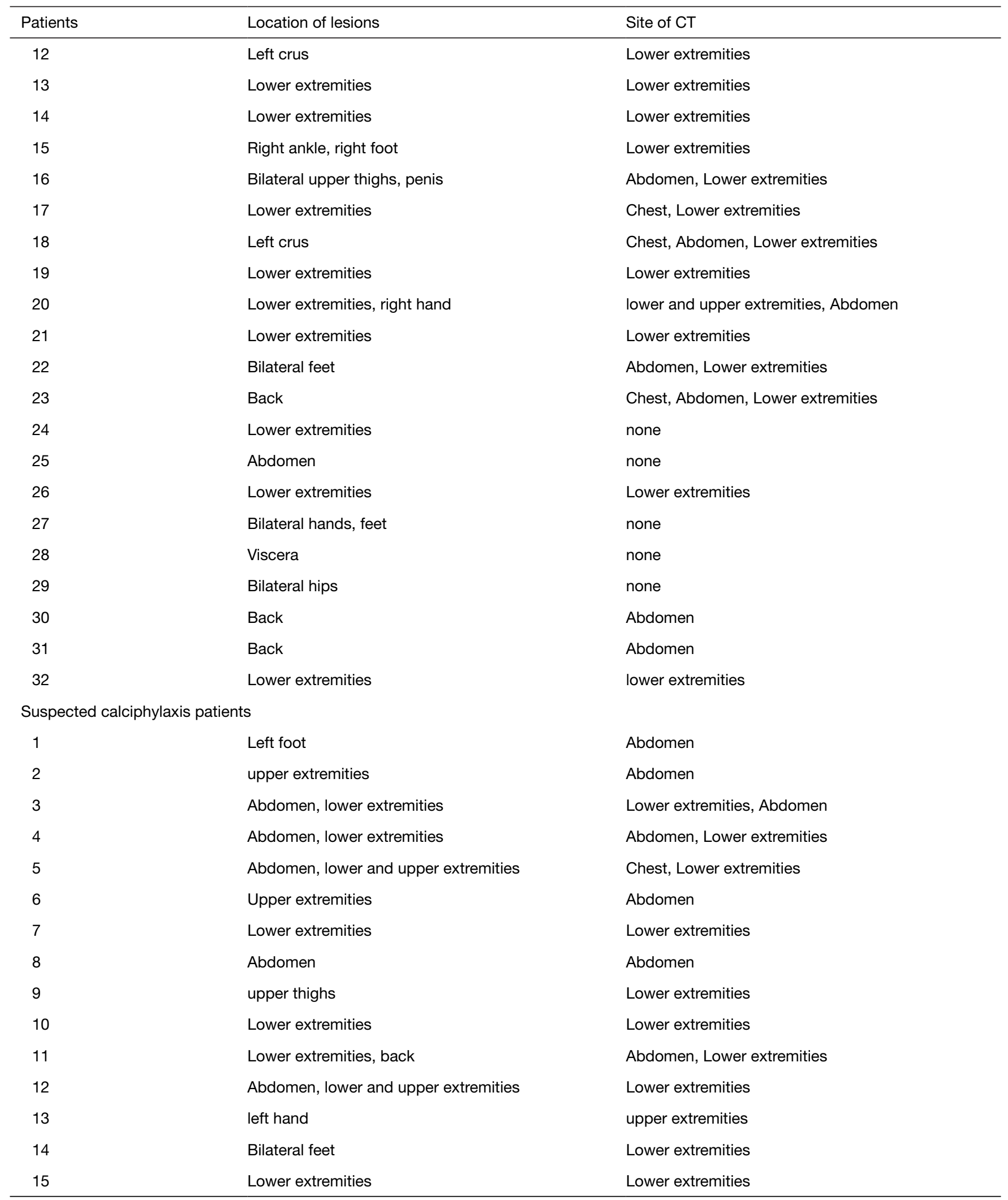



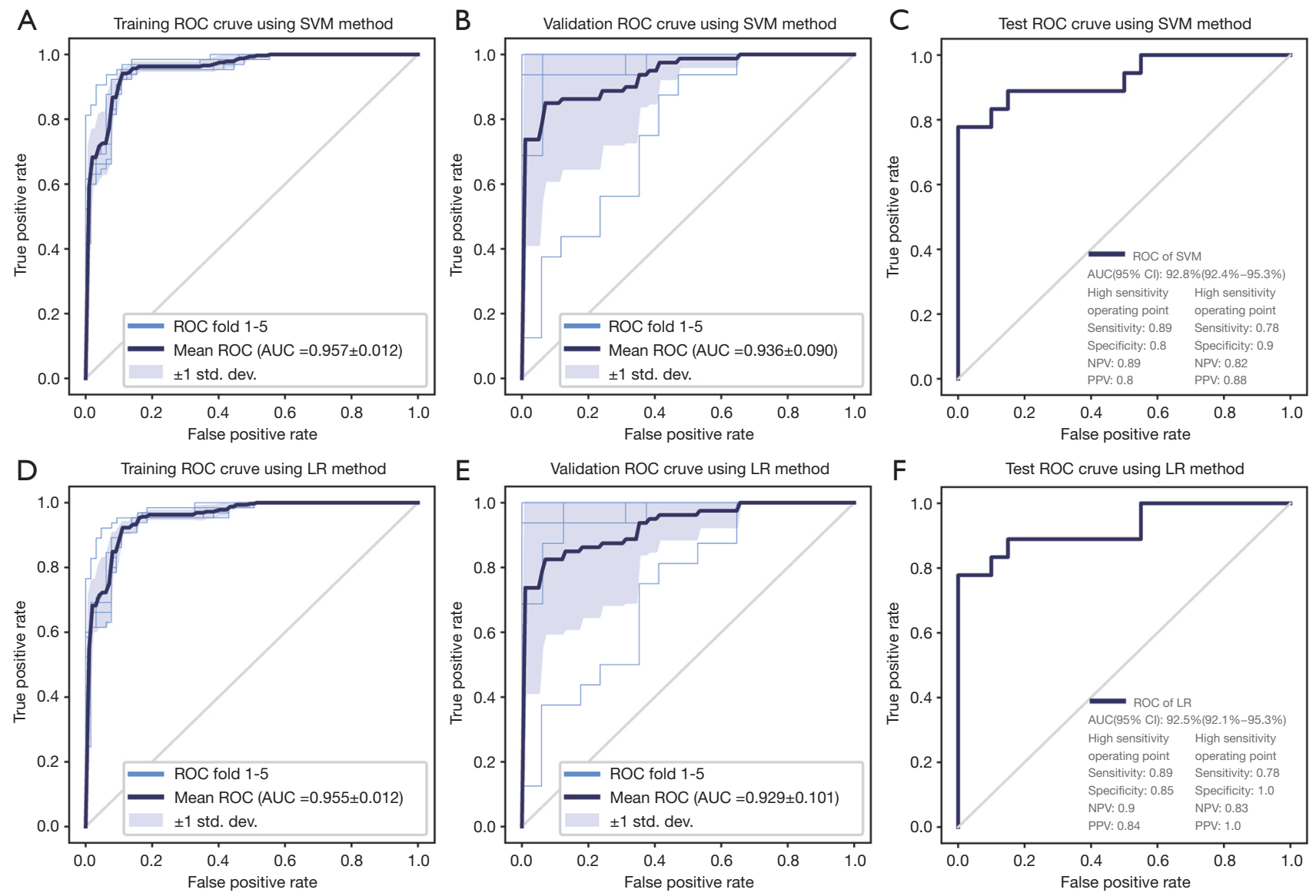

Figure 2 Predictive performance of models in training, inter-validation and test datasets. AUC, areas under the ROC curves; ROC, receiver operating characteristic; LR, logistic regression; SVM, support vector machine; PPV, positive predictive value; NPV, negative predictive value.

Table 3 Predictive performance of radiomic models ${ }^{a}$

\begin{tabular}{|c|c|c|c|c|c|c|c|c|c|}
\hline & \multirow{2}{*}{ AUC (95\% Cl) } & \multicolumn{4}{|c|}{ High sensitivity model } & \multicolumn{4}{|c|}{ High specificity model } \\
\hline & & Sensitivity & Specificity & NPV & PPV & Sensitivity & Specificity & NPV & PPV \\
\hline SVM & $0.93(0.924-0.953)$ & 0.89 & 0.80 & 0.89 & 0.80 & 0.78 & 0.90 & 0.82 & 0.88 \\
\hline LR & $0.93(0.921-0.953)$ & 0.89 & 0.85 & 0.90 & 0.84 & 0.78 & 1.0 & 0.83 & 1.0 \\
\hline
\end{tabular}

${ }^{a}$ Total lesions: 38 (positive: 18/negative: 20). PPV, positive predictive value; NPV, negative predictive value; AUC, areas under the ROC curves; ROC, receiver operating characteristic; LR, logistic regression; SVM, support vector machine.

\section{Discussion}

In this retrospective study, it proposed the radiomics method and demonstrated the effectiveness of the noninvasive machine learning technique in diagnosing skin lesions in patients with calciphylaxis (Figure 2).

The sample comprised 27 histopathological confirmed calciphylaxis patients and 12 histopathological confirmed non-calciphylaxis patients with suspected skin lesions. Given the histopathological examination as the ground truth, the models in this research were confirmed to be stable and could be generalised in the diagnosis of skin lesions in calciphylaxis.

Calciphylaxis diagnosis is challenging. In the test 
Table 4 Predictive performance compared with plain radiography and bone scintigraphy

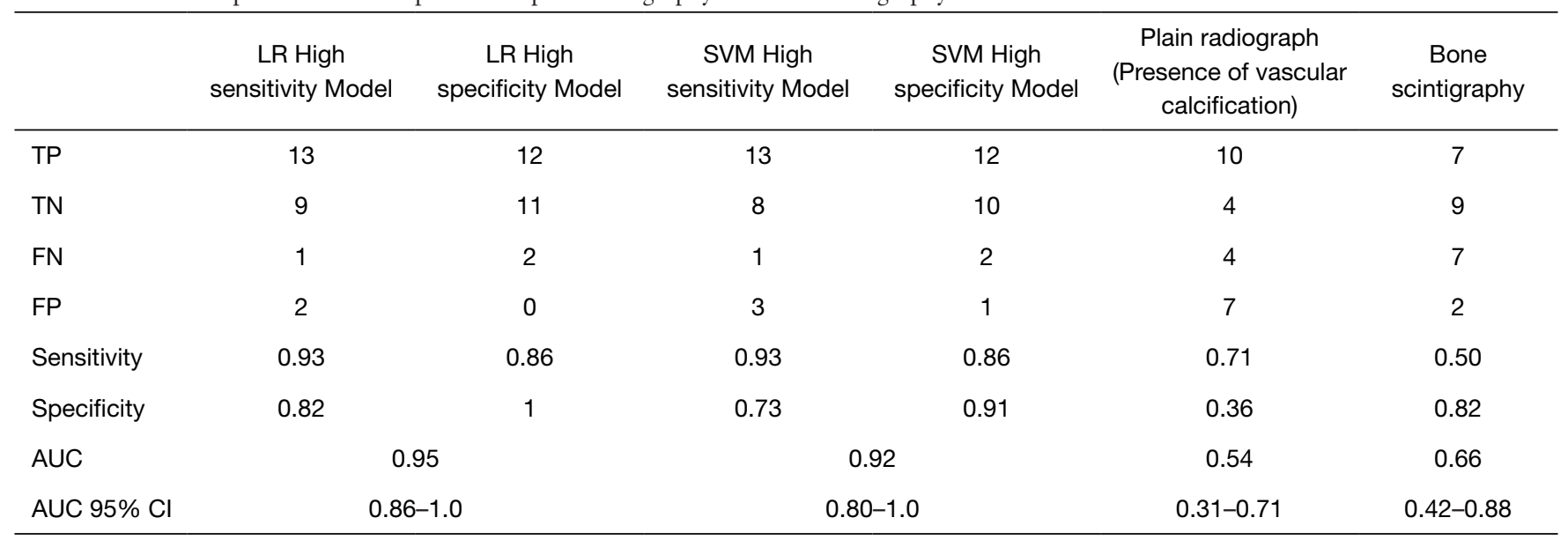

LR, logistic regression; SVM, support vector machine; AUC, areas under the ROC curves; ROC, receiver operating characteristic; TP, true positive; TN, true negative; FN, false negative; FP, false positive.
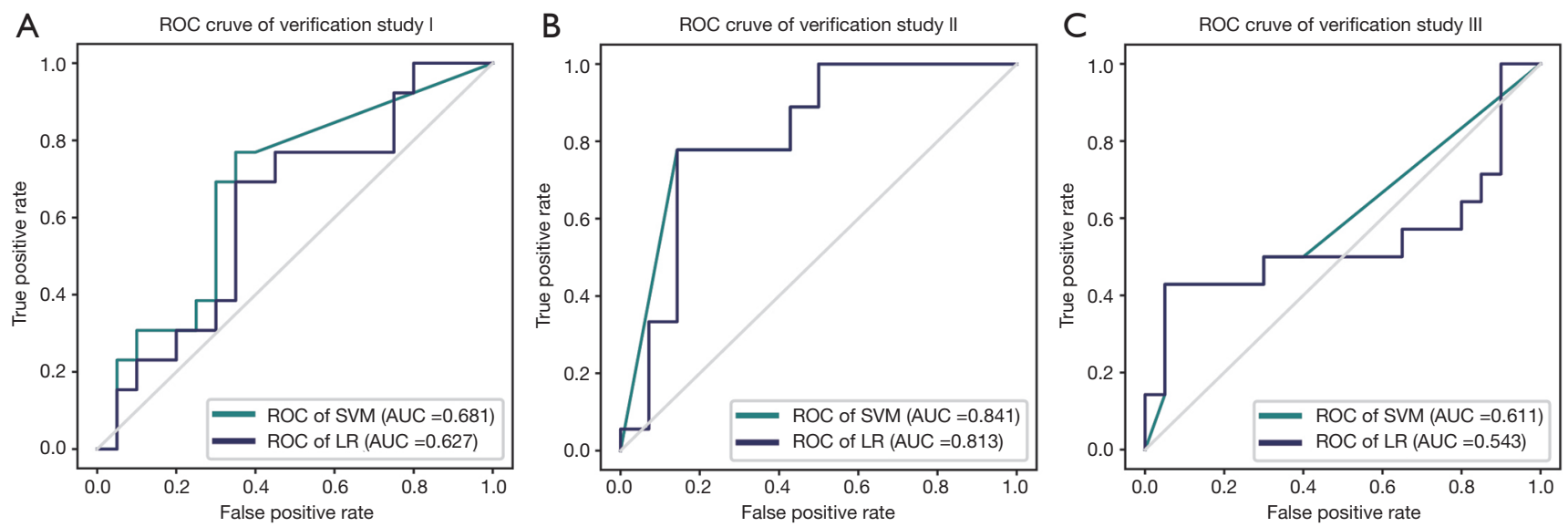

Figure 3 Results of verification studies. AUC, areas under the ROC curves; ROC, receiver operating characteristic.

dataset, radiomic models performed well for diagnosing calciphylaxis in CKD patients, which was better than conventional radiology methods such as plain radiograph and bone scintigraphy (Table 3), the performance of which was similar to that in previous studies $(3,5)$. The exploration of diagnostic models has confirmed the possibility of a noninvasive diagnosis method that might provide clinicians with an alternative when histopathological examination is impossible and thereby reducing the incidence of complications due to invasive operations, especially at the skin lesions (2). However, bone scintigraphy also showed high specificity based on our dataset (0.82), while its sensitivity was 0.5 . Specificity in this study is comparable to that in the research of Paul et al. (0.97) but the sensitivity is much lower (0.89) (5). However, a more accurate noninvasive method can be provided for calciphylaxis diagnosis by combining radiomics methods on CT and bone scintigraphy.

Secondly, the radiomics and machine learning methods were adopted in this study, which are popular in disease diagnosis and prognosis prediction (9). In the models, 50\% of the features included were first-order, while the rest were second-order. Nevertheless, they showed a potential to detect inflammation, fibrosis, and vascularity in adipose tissue, and made the model more robust by being less sensitive to an absolute value $(17,18)$. Similarity in AUC, sensitivity and, specificity for SVM and LR methods demonstrated the robustness of the same radiomic features, 
based on the prior studies' result that the classification method played the most dominant role in the variability of model (15).

In the verification studies, SVM and LR model could not classify suspected lesion and L3 subcutaneous tissue in suspected calciphylaxis patients well. Extracted features might not represent the tissue characteristic in different locations. As manifested in the results of Study II and Study III, our model revealed the possibility that in calciphylaxis patients, tissue in lesion would be distinctive compared to distant tissue like abdomen, and huge differences might not exist between calciphylaxis patients and other CKD patients at the location without skin lesion.

This research has several limitations. It was a retrospective study, and given the low morbidity of calciphylaxis, a small sample size of both patients and controls was enrolled. In the meantime, this research selected a special dataset of negative cases and lesions in training, which might give rise to selection bias. Secondly, this is a single-centre study, and model performance could be different in a large population and other centres. Future studies should explore and validate the noninvasive methods for diagnosis of calciphylaxis in more patients and centres including both imaging-based and serum-based methods.

To the best of our knowledge, this research is the first to develop a radiomics method in calciphylaxis diagnosis. CT radiomics features hidden within skin lesions were extracted and the radiomic model demonstrated preliminary feasibility as a noninvasive technique for calciphylaxis diagnosis in patients with $\mathrm{CKD}$ when invasive procedures are not available.

\section{Acknowledgments}

Funding: This work was supported by grants from the National Natural Science Foundation of China (81570612); National Natural Science Foundation of China (81870497).

\section{Footnote}

Conflicts of Interest: All authors have completed the ICMJE uniform disclosure form (available at https://dx.doi. org/10.21037/qims-20-1211). The authors have no conflicts of interest to declare.

Ethical Statement: The authors are accountable for all aspects of the work in ensuring that questions related to the accuracy or integrity of any part of the work are appropriately investigated and resolved. This retrospective, cohort study was conducted in accordance with the Declaration of Helsinki (as revised in 2013) and was approved by the local institutional ethics review board. Additionally, the need for written informed consent from the participants was waived.

Open Access Statement: This is an Open Access article distributed in accordance with the Creative Commons Attribution-NonCommercial-NoDerivs 4.0 International License (CC BY-NC-ND 4.0), which permits the noncommercial replication and distribution of the article with the strict proviso that no changes or edits are made and the original work is properly cited (including links to both the formal publication through the relevant DOI and the license). See: https://creativecommons.org/licenses/by-nc-nd/4.0/.

\section{References}

1. Nigwekar SU, Thadhani R, Brandenburg VM. Calciphylaxis. N Engl J Med 2018;378:1704-14.

2. Nigwekar SU, Kroshinsky D, Nazarian RM, Goverman J, Malhotra R, Jackson VA, Kamdar MM, Steele DJR, Thadhani RI. Calciphylaxis: Risk Factors, Diagnosis, and Treatment. Am J Kidney Dis 2015;66:133-46.

3. Shmidt E, Murthy NS, Knudsen JM, Weenig RH, Jacobs MA, Starnes AM, Davis MD. Net-like pattern of calcification on plain soft-tissue radiographs in patients with calciphylaxis. J Am Acad Dermatol 2012;67:1296-301.

4. Halasz CL, Munger DP, Frimmer H, Dicorato M, Wainwright S. Calciphylaxis: Comparison of radiologic imaging and histopathology. J Am Acad Dermatol 2017;77:241-246.e3.

5. Paul S, Rabito CA, Vedak P, Nigwekar SU, Kroshinsky D. The Role of Bone Scintigraphy in the Diagnosis of Calciphylaxis. JAMA Dermatol 2017;153:101-3.

6. Colboc H, Moguelet P, Bazin D, Carvalho P, Dillies AS, Chaby G, Maillard H, Kottler D, Goujon E, Jurus C, Panaye $M$, Frochot V, Letavernier E, Daudon M, Lucas I, Weil R, Courville P, Monfort JB, Chasset F, Senet P; Groupe Angio-Dermatologie of the French Society of Dermatology. Localization, Morphologic Features, and Chemical Composition of Calciphylaxis-Related Skin Deposits in Patients With Calcific Uremic Arteriolopathy. JAMA Dermatol 2019;155:789-96.

7. Kramann R, Brandenburg VM, Schurgers LJ, Ketteler M, Westphal S, Leisten I, Bovi M, Jahnen-Dechent W, Knüchel R, Floege J, Schneider RK. Novel insights into 
osteogenesis and matrix remodelling associated with calcific uraemic arteriolopathy. Nephrol Dial Transplant 2013;28:856-68.

8. Antonopoulos AS, Sanna F, Sabharwal N, Thomas S, Oikonomou EK, Herdman L, et al. Detecting human coronary inflammation by imaging perivascular fat. Sci Transl Med 2017;9:eaal2658.

9. Gillies RJ, Kinahan PE, Hricak H. Radiomics: Images Are More than Pictures, They Are Data. Radiology 2016;278:563-77.

10. Lambin P, Leijenaar RTH, Deist TM, Peerlings J, de Jong EEC, van Timmeren J, Sanduleanu S, Larue RTHM, Even AJG, Jochems A, van Wijk Y, Woodruff H, van Soest J, Lustberg T, Roelofs E, van Elmpt W, Dekker A, Mottaghy FM, Wildberger JE, Walsh S. Radiomics: the bridge between medical imaging and personalized medicine. Nat Rev Clin Oncol 2017;14:749-62.

11. McCarthy JT, El-Azhary RA, Patzelt MT, Weaver AL, Albright RC, Bridges AD, Claus PL, Davis MD, Dillon JJ, El-Zoghby ZM, Hickson LJ, Kumar R, McBane RD, McCarthy-Fruin KA, McEvoy MT, Pittelkow MR, Wetter DA, Williams AW. Survival, Risk Factors, and Effect of Treatment in 101 Patients With Calciphylaxis. Mayo Clin Proc 2016;91:1384-94.

12. Liu Y, Zhang X, Xie X, Yang X, Liu H, Tang R, Liu B. Risk factors for calciphylaxis in Chinese hemodialysis patients: a matched case-control study. Ren Fail 2021;43:406-16.

Cite this article as: Yu Q, Liu Y, Xie X, Liu J, Huang S, Zhang X, Ju S. Radiomics-based method for diagnosis of calciphylaxis in patients with chronic kidney disease using computed tomography. Quant Imaging Med Surg 2021;11(11):4617-4626. doi: 10.21037/qims-20-1211
13. Di J, Liu Y, Wang D, Yang M. A Case of Early Calciphylaxis Diagnosed by Bone Scan. Case Rep Med 2020;2020:9526836.

14. van Griethuysen JJM, Fedorov A, Parmar C, Hosny A, Aucoin N, Narayan V, Beets-Tan RGH, Fillion-Robin JC, Pieper S, Aerts HJWL. Computational Radiomics System to Decode the Radiographic Phenotype. Cancer Res 2017;77:e104-7.

15. Parmar C, Grossmann P, Bussink J, Lambin P, Aerts HJWL. Machine Learning methods for Quantitative Radiomic Biomarkers. Sci Rep 2015;5:13087.

16. Chilamkurthy S, Ghosh R, Tanamala S, Biviji M, Campeau NG, Venugopal VK, Mahajan V, Rao P, Warier P. Deep learning algorithms for detection of critical findings in head CT scans: a retrospective study. Lancet 2018;392:2388-96.

17. Oikonomou EK, Williams MC, Kotanidis CP, Desai MY, Marwan M, Antonopoulos AS, et al. A novel machine learning-derived radiotranscriptomic signature of perivascular fat improves cardiac risk prediction using coronary CT angiography. Eur Heart J 2019;40:3529-43.

18. Elshafeey N, Kotrotsou A, Hassan A, Elshafei N, Hassan I, Ahmed S, Abrol S, Agarwal A, El Salek K, Bergamaschi S, Acharya J, Moron FE, Law M, Fuller GN, Huse JT, Zinn PO, Colen RR. Multicenter study demonstrates radiomic features derived from magnetic resonance perfusion images identify pseudoprogression in glioblastoma. Nat Commun 2019;10:3170. 
Supplementary

TRIPOD Checklist: Prediction Model Development and Validation

\begin{tabular}{|c|c|c|c|c|}
\hline Section/Topic & Item & & Checklist Item & Page \\
\hline Title & 1 & $\mathrm{D} ; \mathrm{V}$ & $\begin{array}{l}\text { Identify the study as developing and/or validating a multivariable prediction model, } \\
\text { the target population, and the outcome to be predicted. }\end{array}$ & 1 \\
\hline Abstract & 2 & $\mathrm{D} ; \mathrm{V}$ & $\begin{array}{l}\text { Provide a summary of objectives, study design, setting, participants, sample size, } \\
\text { predictors, outcome, statistical analysis, results, and conclusions. }\end{array}$ & 3 \\
\hline \multirow[t]{2}{*}{$\begin{array}{l}\text { Background and } \\
\text { objectives }\end{array}$} & 3a & $\mathrm{D} ; \mathrm{V}$ & $\begin{array}{l}\text { Explain the medical context (including whether diagnostic or prognostic) and } \\
\text { rationale for developing or validating the multivariable prediction model, including } \\
\text { references to existing models. }\end{array}$ & 5 \\
\hline & $3 b$ & $\mathrm{D} ; \mathrm{V}$ & $\begin{array}{l}\text { Specify the objectives, including whether the study describes the development or } \\
\text { validation of the model or both. }\end{array}$ & 5 \\
\hline Source of data & $4 b$ & $\mathrm{D} ; \mathrm{V}$ & $\begin{array}{l}\text { Specify the key study dates, including start of accrual; end of accrual; and, if } \\
\text { applicable, end of follow-up. }\end{array}$ & $6-7$ \\
\hline \multirow[t]{3}{*}{ Participants } & $5 a$ & $\mathrm{D} ; \mathrm{V}$ & $\begin{array}{l}\text { Specify key elements of the study setting (e.g., primary care, secondary care, } \\
\text { general population) including number and location of centres. }\end{array}$ & $6-7$ \\
\hline & $5 b$ & $\mathrm{D} ; \mathrm{V}$ & Describe eligibility criteria for participants. & $6-7$ \\
\hline & $5 c$ & $\mathrm{D} ; \mathrm{V}$ & Give details of treatments received, if relevant. & NA \\
\hline Outcome & $6 a$ & $\mathrm{D} ; \mathrm{V}$ & $\begin{array}{l}\text { Clearly define the outcome that is predicted by the prediction model, including how } \\
\text { and when assessed. }\end{array}$ & $6-8$ \\
\hline Sample size & 8 & $\mathrm{D} ; \mathrm{V}$ & Explain how the study size was arrived at. & $\begin{array}{l}\text { 6-7 Supplementary } \\
\text { method }\end{array}$ \\
\hline Missing data & 9 & $\mathrm{D} ; \mathrm{V}$ & $\begin{array}{l}\text { Describe how missing data were handled (e.g., complete-case analysis, single } \\
\text { imputation, multiple imputation) with details of any imputation method. }\end{array}$ & NA \\
\hline \multirow{5}{*}{$\begin{array}{l}\text { Statistical } \\
\text { analysis methods }\end{array}$} & $10 a$ & $\mathrm{D}$ & Describe how predictors were handled in the analyses. & $7-11$ \\
\hline & $10 \mathrm{~b}$ & $\mathrm{D}$ & $\begin{array}{l}\text { Specify type of model, all model-building procedures (including any predictor } \\
\text { selection), and method for internal validation. }\end{array}$ & $7-11$ \\
\hline & $10 \mathrm{c}$ & V & For validation, describe how the predictions were calculated. & $7-11$ \\
\hline & $10 d$ & $\mathrm{D} ; \mathrm{V}$ & $\begin{array}{l}\text { Specify all measures used to assess model performance and, if relevant, to } \\
\text { compare multiple models. }\end{array}$ & $7-11$ \\
\hline & $10 \mathrm{e}$ & $\mathrm{V}$ & Describe any model updating (e.g., recalibration) arising from the validation, if done. & NA \\
\hline Risk groups & 11 & $\mathrm{D} ; \mathrm{V}$ & Provide details on how risk groups were created, if done. & NA \\
\hline $\begin{array}{l}\text { Development vs. } \\
\text { validation }\end{array}$ & 12 & V & $\begin{array}{l}\text { For validation, identify any differences from the development data in setting, eligibility } \\
\text { criteria, outcome, and predictors. }\end{array}$ & $7-11$ \\
\hline
\end{tabular}


Results

Participants

Model

development

Model

specification

Model

performance

Model-updating

Discussion

Limitations

Interpretation

Implications

Other information

Supplementary information

Funding
$\mathrm{D} ; \mathrm{V}$ Describe the flow of participants through the study, including the number of participants with and without the outcome and, if applicable, a summary of the follow-up time. A diagram may be helpful.

13b $\quad \mathrm{D} ; \mathrm{V}$ Describe the characteristics of the participants (basic demographics, clinical features, available predictors), including the number of participants with missing data for predictors and outcome.

13c V For validation, show a comparison with the development data of the distribution of important variables (demographics, predictors and outcome).

14a D Specify the number of participants and outcome events in each analysis.

14b D If done, report the unadjusted association between each candidate predictor and outcome.

15a D Present the full prediction model to allow predictions for individuals (i.e., all regression 12-14 Supplementary coefficients, and model intercept or baseline survival at a given time point).

Table

15b D Explain how to the use the prediction model.

$12-14$

$16 \mathrm{D} ; \mathrm{V}$ Report performance measures (with Cls) for the prediction model.

$17 \mathrm{~V}$ If done, report the results from any model updating (i.e., model specification, model performance).

12 Table 1 Figure1

NA

12-14, Table $3 \& 4$

Figure $2 \& 3$

NA

16

$14-16$

14-16

19b D;V Give an overall interpretation of the results, considering objectives, limitations, results from similar studies, and other relevant evidence.

$20 \mathrm{D} ; \mathrm{V}$ Discuss the potential clinical use of the model and implications for future research.

$14-16$

$21 \mathrm{D} ; \mathrm{V}$ Provide information about the availability of supplementary resources, such as study protocol, Web calculator, and data sets.

Supplementary material

17

*Items relevant only to the development of a prediction model are denoted by $\mathrm{D}$, items relating solely to a validation of a prediction model are denoted by $\mathrm{V}$, and items relating to both are denoted $\mathrm{D} ; \mathrm{V}$. We recommend using the TRIPOD Checklist in conjunction with the TRIPOD Explanation and Elaboration document. 


\section{Method}

\section{Diagnosis protocol of calciphylaxis}

Calciphylaxis diagnosis protocol was based on "Criteria for Diagnosis of Calciphylaxis" described in McCarthy et al. (11). One patient with risk factors and clinical conditions would receive multidisciplinary discussion including nephrology, dermatology, radiology, and pathology departments with experienced doctors.

In this study, we retrospectively reviewed our institutional datasets for patients with initial diagnosis of calciphylaxis. If one finally diagnosed as calciphylaxis, we included as calciphylaxis patients. If one with possible calciphylaxis but finally ruled out, we included as suspected calciphylaxis patients. Viscera calciphylaxis was not included in analysis. Finally, 32 patients were diagnosed according to reported diagnosis protocol(calciphylaxis) (11), and 15 patients were identified as suspected calciphylaxis from October 1, 2017, to November 30, 2019.

In the next step, we searched CT scan data involving lesion location. For example, if one patient had ulceration in right leg, we would find lower extremities non-contrast CT images in Picture Archiving and Communication Systems. All CT scan followed routine CT scan protocols. All images and masks were resampled to form isotropic voxels of unit dimension with 1 voxel corresponding to $1 \mathrm{~mm}^{3}$ to ensure comparability. By centering the image at the mean with a standard deviation and recharting the histogram to conform to $1 \pm 3 \mathrm{r}$ (l: the average gray level within the VOI; r: the gray-level standard deviation), image normalization was realized.

\section{Sample size consideration}

For training, we used 70\% (19 patients with 40 lesions) randomly chosen calciphylaxis patients and all CKD-non-calciphylaxis patients (41 patients with 82 patches) to form training cohort. To balance the case-to-noncase ratio as 1:1, we used SMOTE method to up-sample the calciphylaxis cases, which is a re-sampling technique commonly used in datasets. We applied 5-fold cross-validation on training data set to prove model performance. Eight features were selected after PCC and Relief, which making an event-per-predictor ratio $>20$. Therefore, we believed that there was no big concern on the overfitting issue of our model at this sample size.

For test, we used method introduced by Shein-Chung Chow and colleagues (19).

$$
N_{\text {positive }}=N_{\text {negative }}=2\left(\sigma \frac{z_{\alpha / 2}+z_{\beta}}{\mu_{\text {positive }}-\mu_{\text {negative }}}\right)^{2}
$$

where, $\mathrm{N}$ is the sample size for the validation group. Desired two-sided significance level of $\alpha=0.05\left(\mathrm{z}_{\alpha 2}=1.96\right)$ and power of $1-\beta=95 \%\left(\mathrm{z}_{\beta}=1.64\right)$.

LR model: The sample sizes in the training groups were $n_{\text {positive }}=82$ (SMOTE) and $n_{\text {negative }}=82$, with means of $\mu_{\text {positive }}=0.3927$ and $\mu_{\text {negative }}=0.6075$ respectively, and with a standard deviation of $\sigma=0.1369$. The minimum number of validation samples:

$$
N_{\text {positive }}=N_{\text {negative }}=2\left(\sigma \frac{z_{\alpha / 2}+z_{\beta}}{\mu_{\text {positive }}-\mu_{\text {negative }}}\right)^{2} \approx 11
$$

SVM model: The sample sizes in the training groups were $\mathrm{n}_{\text {positive }}=82$ (SMOTE) and $\mathrm{n}_{\text {negative }}=82$, with means of $\mu_{\text {positive }}=0.1688$ and $\mu_{\text {negative }}=0.8133$ respectively, and with a standard deviation of $\sigma=0.3948$. The minimum number of validation samples:

$$
N_{\text {positive }}=N_{\text {negative }}=2\left(\sigma \frac{z_{\alpha / 2}+z_{\beta}}{\mu_{\text {positive }}-\mu_{\text {negative }}}\right)^{2} \approx 10
$$

Test dataset in our study included 18 calciphylaxis-positive and 20 calciphylaxis-negative lesions, which exceeded the minimum required sample sizes.

\section{References}

19. Chow S, Shao J, Wang H. Sample size calculations in clinical research. 2nd Ed. Chapman \&Hall//CRC Biostatistics Series 2008. 
Table S1 Features and coefficients of models

\begin{tabular}{lcc}
\hline Features & Coef in model (SVM) & Coef in model (LR) \\
\hline wavelet-LLH_glszm_GrayLevelVariance & 1.330 & 1.187 \\
wavelet-LLH_glcm_Imc2 & 2.930 & 2.524 \\
wavelet-HLH_firstorder_Skewness & 0.703 & 0.578 \\
wavelet-HHH_glszm_LargeAreaLowGrayLevelEmphasis & 0.594 & 0.316 \\
original_firstorder_Kurtosis & -0.300 & 0.148 \\
wavelet-LLL_firstorder_90Percentile & 1.982 & 1.485 \\
wavelet-LHH_firstorder_Median & 3.460 & 2.168 \\
wavelet-LLH_glcm_Imc1 & -4.422 & -3.164 \\
\hline
\end{tabular}

Coef, coefficients.

Table S2 Results of Delong tests

\begin{tabular}{lcc}
\hline ROC 1 & ROC 2 & p-value \\
\hline LR & Bone scintigraphy & $<0.01$ \\
LR & Plain radiograph & $<0.01$ \\
SVM & Bone scintigraphy & 0.02 \\
SVM & Plain radiograph & $<0.01$ \\
\hline
\end{tabular}

LR, Logistic Regression; SVM, Support Vector Machine; AUC, Areas under the ROC curves; ROC, Receiver operating characteristic. 